\title{
IRRATIONAL NODE DETECTION IN MULTIHOP CELLULAR NETWORKS USING ACCOUNTING CENTER
}

\author{
D Krishna Mohan ${ }^{1}$, B. Lalitha ${ }^{2}$ \\ ${ }^{1}$ Post graduate, ${ }^{2}$ Assistant professor, Department of computer science and engineering, \\ JNTU Ananthapur, Andhra Pradesh, India
}

\begin{abstract}
In multihop cellular networks mobile nodes typically transmit packets during intermediate mobile nodes for enhancing recital. Stingy nodes typically don't collaborate that incorporates a negative result on the network fairness and recital. A fair, inexpensive and best incentive mechanism by Selfish Node Detection (FESCIMbySND) has been projected to stimulate the mobile node's cooperation. Hashing operations area unit employed in order to extend the safety. Trivial Hash perform has been wont to improve end-to-end delay and outturn. Additionally Cyclic Redundancy Check Mechanism has been used to spot the ridiculous nodes that involve themselves in sessions with the intention of dropping the in sequence packets. Moreover, to cut back the impact at the Accounting Center a Border node has been commend the task of propose the checks employing a digital signature.
\end{abstract}

Keywords: Border Node Mechanism, Cyclic Redundancy Check, Selfish nodes, Trivial Hash Function ***.

\section{INTRODUCTION}

Reputation-based and incentive mechanisms are projected to avoid stinginess attacks. For reputation-based mechanisms the nodes sometimes monitor the transmissions of their neighbors to create certain that the neighbors relay different nodes' traffic, and thus, narcissistic nodes are often known and chastised. For incentive mechanisms, packet relay may be a service not AN obligation. The supply and destination nodes pay credits (or virtual currency) to the intermediate nodes for relaying their packets. Credits will stimulate the nodes' cooperation by proving that it's a lot of useful for the nodes to collaborate than behaving egotistically.

A fair and economical incentive mechanism has been projected to stimulate the node cooperation. Hashing operations square measure employed in the ACK packets to cut back the quantity of public-key- cryptography operations.

Trivial Hash operate has been accustomed cut back the quantity of public key cryptanalytic operations. Additionally it additionally has the advantage that it will cut back the computation value. Cyclic Redundancy Check Mechanism has been utilized to spot the irrational nodes that involve themselves in sessions with the intention of dropping the information packets to launch Denial-of-Service attacks.

Moreover, reducing the overhead of the payment checks is important for the economical implementation of the motivation mechanism thanks to the massive variety of payment transactions.
To change this, rather than permitting every of the intermediate nodes to submit checks one by one for expense a Border node has been commend the task of submitting the checks employing a digital signature. in depth analysis and simulations demonstrate that the projected system allows economical knowledge transfer by police investigation irrational nodes

The network potency has been considerably impermanent by reducing the overhead of check submission victimization Border node mechanism. What is more Trivial Hash operate has considerably reduced the value of computing hash functions while not compromising the message integrity.

\section{LITERATURE REVIEW}

A secure mechanism is bestowed [1] to stimulate finish users to stay their devices turned on, to refrain from overloading the network, and to avoid change of state aimed toward changing the device into a "selfish" one. A regionally Aware name system has been planned [2] that addresses inconsiderate behavior by exploitation regionally out there data. an summary of security issues [3] for mobile impromptu networks distinctive the threats on basic mechanisms and on security mechanisms is mentioned. A micro-payment theme has been planned [4] for multi-hop cellular networks that encourage collaboration in packet forwarding by lease users take pleasure in relaying others' packets. A protocol [5] is bestowed for routing in impromptu networks that uses Dynamic supply Routing. Multihop Cellular Network (MCN), for wireless communications [6] preserves the good thing about typical Single Hop Cellular Networks (SCN) wherever the service infrastructure is made by fastened bases, and it conjointly 
incorporates the flexibleness of ad-hoc networks wherever wireless transmission through mobile stations in multiple hops is allowed. In multihop cellular networks, the mobile nodes typically relay others' packets [7] for enhancing the network performance and readying. a good and economical incentive mechanism-FESCIM has been planned to stimulate the node cooperation. 2 techniques are planned that improve output in Associate in Nursing Ad-hoc network [8] within the presence of nodes that comply with forward packets however fail to try to to thus. there's a watchdog that identifies misbehaving nodes and there's a pathrater that helps routing protocols avoid these nodes. A simulation study is applied [9] that identifies security problems that square measure specific to Manet which illustrate the results of these threats on network performance once the DSR routing protocol is employed. A multi-party micropayment [10] theme has been planned that enables all parties concerned during a decision to be paid in period of time. Multi-hop cellular networks (also known as hybrid networks) seem to be a capable combination of the dynamics of mobile unprepared networks and therefore the dependableness of infrastructure wireless networks [11]. These hybrid networks provide many blessings for users yet as operators.

A Cooperation and Accounting Strategy for Hybrid Networks referred to as CASHnet has been projected that stimulates cooperation among nodes by creating it a rewardable different to stinginess. Our theme incorporates security design, that relies on public key cryptography and uses digital signatures and certificates. Today's public Wireless LANs area unit restricted to hotspots [12]. With this technology, suppliers will solely target a tiny low audience and successively charge high costs for his or her service to get revenue. Also, suppliers cannot react befittingly to dynamic changes within the demand. Therefore, a cooperation and accounting theme has been that introduces financial rewards. CASHnet is compared with the Nuglet theme victimization simulations below the factors of network liveliness, overhead, and packet drop reasons yet as income. CASHnet, uses a extremely suburbanized accounting and security design .It permits ungenerous nodes and supports price sharing between sender and receivers situated in numerous sub networks. Integrated Cellular And unintended Relaying Systems (iCAR) [13] could be a new wireless system design supported the mixing of cellular and fashionable unintended relaying technologies. It addresses the congestion drawback as a result of unbalanced traffic in an exceedingly cellular system and provides ability for heterogeneous networks. the correct functioning of mobile unintended networks depends on the hypothesis that every individual node is prepared to forward packets for others [14].To address this drawback, we tend to propose a credit-based Secure Incentive Protocol (SIP) to stimulate cooperation among mobile nodes with individual interests. SIP will be enforced in an exceedingly totally distributed manner and doesn't need any pre-deployed infrastructure. Faerie [15], a simple, cheat-proof, credit primarily based system is projected for exciting cooperation among ungenerous nodes in mobile unintended networks.

\section{EXISTING SYSTEM}

In multihop cellular networks, the mobile nodes typically relay alternative node's packets for enhancing the network performance and readying. As a primary step to the present method in FESCIM [7], a route is about up between supply and destination by the Route Discovery section. throughout this the sender can send a Route Request Packet to its neighbors that contain the destination address. The neighbors successively append their own address to the Route Request Packet and air it. This method continues till the Route Request Packet reaches the destination. The destination can ensure this route by a Route Reply Packet. the info packet being sent are encrypted by mistreatment hashing operation. Additionally hashing method also will scale back the quantity of public-key- science operations. Upon receiving the Route Reply Packet, every intermediate node relays the packet.

However, self-serving nodes typically don't work however build use of alternative nodes for relaying the packets. Such nodes are known and punished. Once fortunate information transfer the co-operating nodes can submit a check to the Accounting Center for compensation of resources. The Accounting Center can then distribute the credits to the cooperative nodes once verification of the checks.

\section{PROPOSED SYSTEM}

From the studies conducted over the years it's been clear that in information transfer some delay still exists. Ungenerous behavior among nodes is found to be one necessary reason for this delay in information delivery. Numerous techniques are adopted to scale back this ungenerous behavior and enhance cooperation among intermediate nodes however it's been distinguished that some wrongful conduct still exists because of the presence of irrational nodes within the network. The prevailing system will avoid stinginess attacks however it cannot establish the illogical nodes that involve themselves in sessions with the purpose of dropping the information packets. Techniques may well be adopted to seek out such irrational nodes.

Previously hashing operations are utilized to secure the information being transferred over the network. However but hashing operations conjointly create to some machine delay.

This price of computation may well be reduced exploitation higher hashing techniques.

Moreover every of the cooperating nodes that have participated in successful information transfer submit checks to the Accounting Center for compensation of resources. this will end in congestion at the Accounting Center. Steps may well be taken to scale back this hold up. 
The planned system aims at overcoming of these draw backs.

- $\quad$ Reduces time delay concerned in information transfer by distinctive irrational nodes.

- $\quad$ Reduces the machine price of hashing

- $\quad$ Reduces the hold up at the Accounting Center

\subsection{CRC- Cyclic Redundancy Check}

Cyclic Redundancy Check are often used for handling irrational nodes. Every information packet to be transmitted is appended with a check and this check is calculated at each hop of knowledge transfer. If there's a distinction within the check worth there's an information loss so specific node is discerned and blocked. Such nodes are irrational nodes. This can facilitate avoid information loss and guarantee high outturn and cut back finish to finish delay.

\subsection{Trivial Hash Function}

Trivial Hash perform has been wont to scale back the price of computing the hash perform. If the data point to be hashed is tiny enough, one will use the data point itself because the hashed price. the price of computing this "trivial" hash perform is effectively zero. This hash perform is because it maps every input to a definite hash price. Associate degree index table is maintained that offers alternate type of the character Invalid knowledge values could also be left indefinable within the table, or mapped to some acceptable "null" price.

\subsection{Border Node Mechanism}

To modify congestion at the Accounting Center a border node is entrusted the responsibility of check submission. All the intermediate cooperating nodes can deliver the checks to the current Border Node. This Border Node can then generate a final check and secure it with a digital signature and send it to the Accounting Center. This can guarantee less congestion at the Accounting Center and facilitate modify the bottleneck downside.

\section{RESULT AND DISCUSSION}

Java Network machine (JNS) is employed for simulation discovered. JNS may be a common network simulation tool used for network research lab experimentation and analysis. JNS provides network performance metrics at varied abstraction levels like Network, sub-network, Node and an in depth packet trace. Mistreatment JNS modeling and simulation services area unit provided in an exceedingly kind of networking technologies and protocols. Results obtained area unit as shown below. Table I, II, III shows variation in turnout, finish to finish delay and packet loss for twelve nodes severally. Equally the graphs obtained for these variations also are shown below in Figures two, 3, four severally.

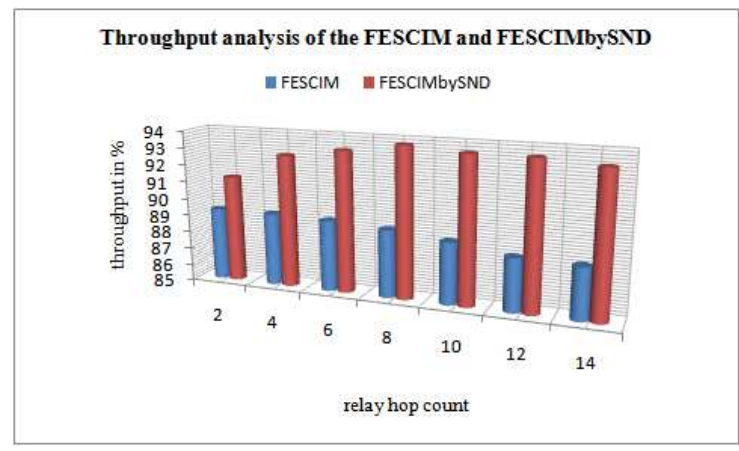

Fig 1: Comparison of throughput

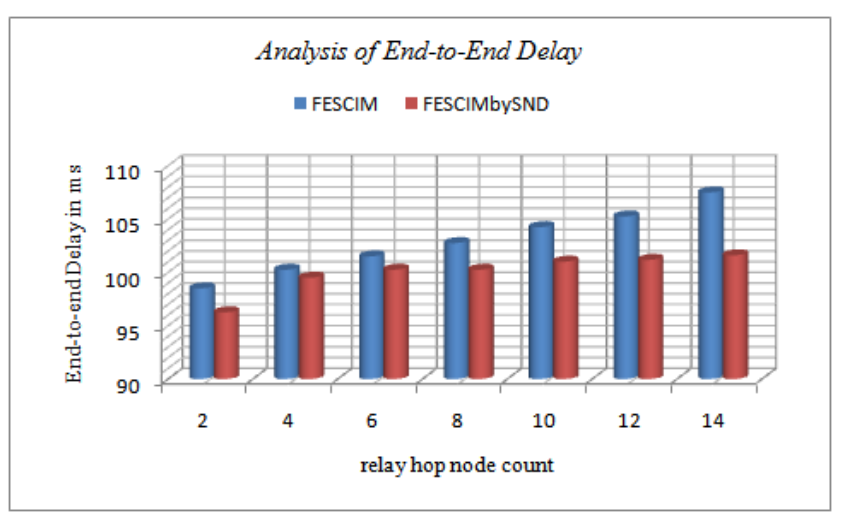

Fig 2: Comparison of delay

\section{CONCLUSIONS}

A finest and secure transmission mechanism has been projected for Multihop Cellular Networks. Irrational nodes have been originate out thereby increasing throughput by $9.4 \%$ and plummeting end to end delay by $1.6 \%$. Extensive simulations show that the projected mechanism achieves enhanced transmission by reducing the packet loss by $9 \%$.

\section{REFERENCES}

[1] X. Li, B. Seet, and P. Chong, "Multihop Cellular Networks: Technology and Economics,"Computer Networks,vol. 52, no. 9, pp. 1825-1837, June 2008.

[2] C. Gomes and J. Galtier, "Optimal and Fair Transmission Rate Allocation Problem in Multi-Hop Cellular Networks,'Proc. Int'l Conf. Ad-Hoc, Mobile and Wireless Networks,pp. 327-340, Aug. 2009.

[3] G. Shen, J. Liu, D. Wang, J. Wang, and S. Jin, "MultiHop Relay for Next-Generation Wireless Access Networks,’Bell Labs Technical J., vol. 13, no. 4, pp. 175-193, 2009.

[4] R. Schoenen, R. Halfmann, and B. Walke, "MAC Performance of a 3GPP-LTE Multihop Cellular Network," Proc. IEEE Int'l Conf. Comm. (ICC),pp. 4819-4824, May 2008. 
[5] G. Marias, P. Georgiadis, D. Flitzanis, and K. Mandalas, "Cooperation Enforcement Schemes for MANETs: A Survey," J. Wireless Comm. and Mobile Computing,vol. 6, no. 3, pp. 319-332, 2006.

[6] C. Song and Q. Zhang, "OMH-Suppressing Selfish Behavior in Ad Hoc Networks with One More Hop,"Mobile Networks and Applications,vol. 14, no. 2, pp. 178-187, Feb. 2009.

[7] D. Djenouri and N. Badache, "On Eliminating Packet Droppers in MANET: A Modular Solution,"Ad Hoc Networks,vol. 7, no. 6, pp. 1243-1258, Aug. 2009.

[8] G. Bella, G. Costantino, and S. Riccobene, "Evaluating the Device Reputation Through Full Observation in MANETs,"J. Information Assurance and Security,vol. 4, no. 5, pp. 458-465, Mar. 2009.

[9] Y. Zhang, W. Lou, and Y. Fang, "A Secure Incentive Protocol for Mobile Ad Hoc Networks,"ACM Wireless Networks,vol. 13, no. 5, pp. 569-582, Oct. 2007.

[10] A. Weyland, "Cooperation and Accounting in Multi-Hop Cellular Networks," PhD thesis, Univ. of Bern, Nov. 2005.

[11] A. Weyland, T. Staub, and T. Braun, "Comparison of MotivationBased Cooperation Mechanisms for Hybrid Wireless Networks," J. Computer Comm.,vol. 29, pp. 2661-2670, 2006.

[12] M. Mahmoud and X. Shen, "Stimulating Cooperation in MultiHop Wireless Networks Using Cheating Detection System,"Proc. IEEE INFOCOM,pp. 776-784, Mar. 2010.

[13] J. Pan, L. Cai, X. Shen, and J. Mark, "Identity-Based Secure Collaboration in Wireless Ad Hoc Networks,"Computer Networks, vol. 51, no. 3, pp. 853865, 2007.

[14] N. Salem, L. Buttyan, J. Hubaux, and M. Jakobsson, "Node Cooperation in Hybrid Ad Hoc Networks,"IEEE Trans. Mobile Computing,vol. 5, no. 4, pp. 365-376, Apr. 2006.

[15] H. Janzadeh, K. Fayazbakhsh, M. Dehghan, and M. Fallah, "A Secure Credit-Based Cooperation Stimulating Mechanism for MANETs Using Hash Chains,"Future Generation Computer Systems,vol. 25, no. 8, pp. 926934, Sept. 2009.

[16] Nat'l Inst. of Standards and Technology (NIST), "Recommendation for Key Management - Part 1: General (Revised)," Special Publication 800-57 200, 2007.

[17] W. Dai, "Crypto++ Library 5.6.0," http://www.cryptopp.com, 2011.

[18] N. Potlapally, S. Ravi, A. Raghunathan, and N. Jha, "A Study of the Energy Consumption Characteristics of Cryptographic Algorithms and Security Protocols," IEEE Trans. Mobile Computing, vol. 5, no. 2, pp. 128143, Mar./Apr. 2006. 